\title{
Application of Cheongsam Display in the Museum Based on Virtual Reality Technology
}

\author{
Wenli Lu, and Xiangyang Bian ${ }^{a}$ \\ College of Fashion and Design, Donghua University, Shanghai, China
}

\begin{abstract}
The wide application of Virtual Reality technology has realized the virtualization of display in the museum and created a new form of interaction between visitors and exhibits. Cheongsam is the product of innovative integration of Chinese traditional dress and Western dress. In this paper, we take " Orange silk Qipao with velvet pattern " which exhibits in Shanghai Museum of textile and costume as an example, take the immersive Virtual Reality System as the carrier to introduce the construction process of Cheongsam Virtual Reality System.
\end{abstract}

\section{Introduction}

In recent years, the development of Virtual Reality technology has attracted the attention of various industries. Museums are also gradually trying to integrate new technologies with cultural relics. The museums' Virtual Exhibition which is based on high technology achieves the visitors' real experience in the virtual scene. In this way, the simulation of museum artefacts based on computer technology is a breakthrough in exploring the history of human civilization. This article takes the cheongsam which is in Shanghai Museum of textile and costume as an example to build a virtual exhibition system. Therefore, it provides a new form for mankind to understand history and explore history.

\section{3D modelling of model}

\subsection{D model of characters and Cheongsam}

Characters and cheongsams are similar in their production techniques. Due to the special attributes of cheongsam, the cheongsam's modelling needs to completely restore in details. The authenticity of cultural relics also asks designers to have a strict spirit of recovery in technology. This is also the main feature of Virtual Display of Museum costumes.

\subsubsection{D modelling of characters}

According to the size of cheongsam, it's analysed in character and we get the human data of the figures

\footnotetext{
${ }^{a}$ Corresponding author: isscatherine@hotmail.com
}

(Table 1). Based on the data, the character is created by MAYA. The process of character was divided into four parts: character polygon modelling, skin texture giving, model UV mapping and texture mapping.

Table 1. the date of the Model

\begin{tabular}{|c|c|c|c|c|}
\hline Data & Height & Hipline & Bust & Waist \\
\hline $\begin{array}{c}\text { Number } \\
(\mathrm{cm})\end{array}$ & 165 & 83 & 73 & 62 \\
\hline
\end{tabular}

The character's model needs to be put on clothes, so we don't describe it detailedly, especially the structure of character. The production of hair style is the most critical. According to the old photos which was in the Republic of China, the elements of hair style are extracted to complete the modelling and collocation (Figure 1).
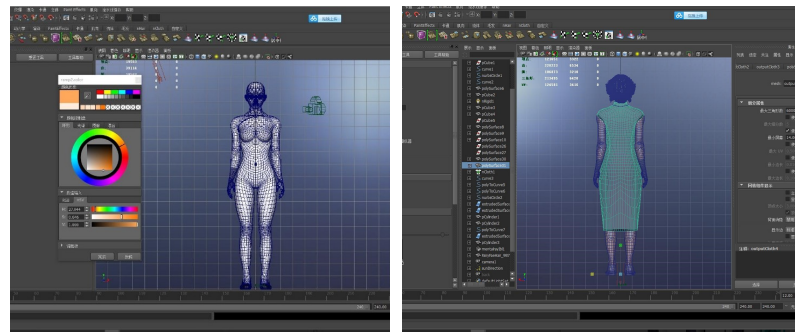

Figure 1. Character model

Figure 2. Cheongsam model

\subsubsection{D modelling of Cheongsam}

At the beginning of modelling, Cheongsam was analysed it in structures and materials, so as to improve 
the authenticity of the model (Figure 2). There are three buttons in this cheongsam, the neckline of the cheongsam and the underside. The large flower pattern on the velvet material is printed on the orange silk. Above characteristics, polygonal modelling is used to restore the style, such as buttons and other structures of object.

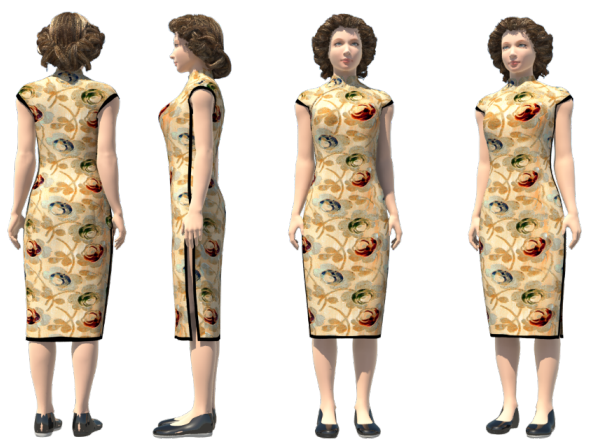

Figure 3. The render of the model

With the help of Photoshop and 3D Paint Tool, the UV map and the texture map are given after completed model. The texture processing determines the authenticity of the model to a certain extent. Mental Ray is used to render the adjustment model continuously, so as to further simulate the softness of cheongsam fabric, and finally achieve the production of characters and cheongsam models (Figure 3 ).

\subsection{D Model of Environmental Scene}

Dress has always been an occasion since ancient times. Cheongsam is also the same as it. Different types of cheongsams have unique characteristics on occasions. The young woman dressed in "Orange silk Qipao with velvet pattern" went through the lively and bustling Nanjing Road, showing visitors the life situation of 1930s.

The young woman turned through the flourishing Nanjing Road into the Paramount, extracting the two parts of Nanjing Road and the Paramount as the core of the big scene. The four major department stores (Xianshi Department Store, Yongan department Store, Xinxin Department Store and Daxin Department Store) and the Paramount consist of five elements of the scene(Figure 4).

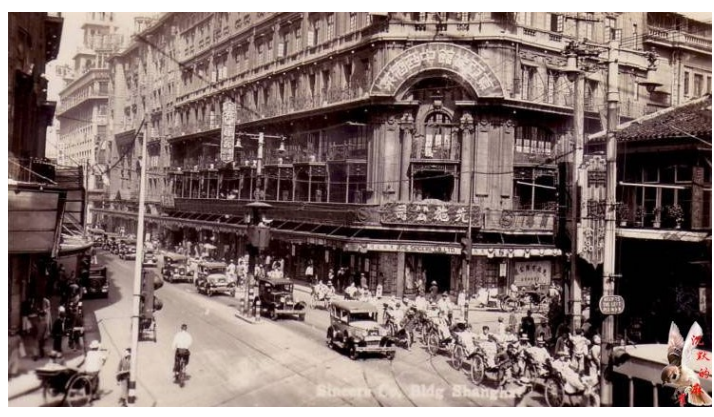

Figure 4. Nanjing Road in 1930s

Different from the cheongsam modelling, the modelling of Nanjing Road is a multi-body combination model. The whole scene can be divided into the main model and the auxiliary model, and the auxiliary buildings are gradually added after the individual main body is built, including the stores or houses which connect the four major department stores and the Paramount, as well as the streetcar tracks, vehicles and pedestrians on the street. Because the living environment of the Republic of China is historical, designers can't set up objects that violate history with their own preferences.

The scene is night view so that lighting is an important and complex part in the whole picture. Fantastic lighting is helpful to unify the independent buildings to form a scene setting atmosphere. It is beneficial to open space relations, to display the bright and dark relations of the environment, to enhance the authenticity of the picture, to enrich the diversity of colours, and to strengthen the sense of volume of the objects. Finally, above these points, we render the output of the model and fine tune it according to the environment (Figure 5).

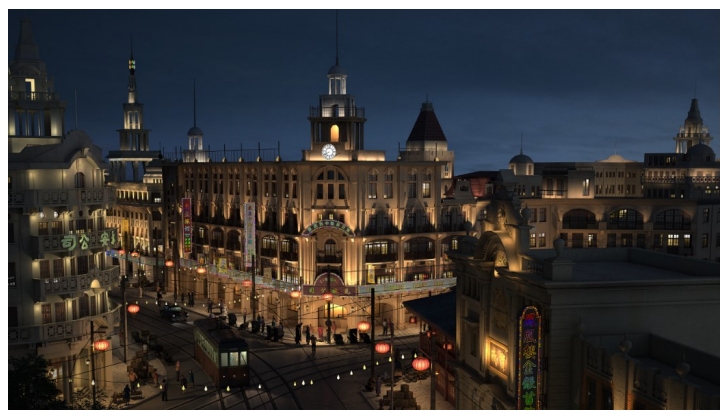

Figure 5. The render of the Environmental Scene

\section{The auxiliary elements of Virtual Display System}

In addition to the two main bodies, cheongsam and scenes, we also need to add interface design, including exhibition information transmission, environmental sound and other auxiliary elements.

The part of exhibition information mainly explains the contents of Cheongsam in the form of text and audio. Text is one of the essential elements in Museum 
exhibition. In the virtual display system of the cheongsam, the text should not be too many complicated languages. Only time and name of Cheongsam are better, 1930s, Orange silk Qipao with velvet pattern. They make the audience understand the exhibits easily. Audio can easily explain the story of cheongsam and help visitors to understand the history of the exhibits and their production technique and application better.

Table 2. The auxiliary elements

\begin{tabular}{|c|c|}
\hline Text & $\begin{array}{c}\text { 1930s, Orange silk Qipao with velvet } \\
\text { pattern; }\end{array}$ \\
\hline Audio & Whistle; Peddle; \\
\hline Music & Rose, Rose, I love you! \\
\hline
\end{tabular}

The environmental sounds are infectious and they can interpret scenarios better in scenes. The figure in table 2 shows that we also can add whistle, peddle and the voice from people into application. The scene of Nanjing Road is in a complex commercial street. It will be integrated into the voice of life. The music coming from the Paramount will be added to the scene, such as "Rose, Rose, I love you!" and other classic music in 1930s.

\section{The application of Virtual Display System}

The Virtual Display System of cheongsam has two kinds of output forms: mobile terminal and PC terminal. Shanghai Museum of textile and costume will develop these two output forms as the same time, but in this paper taking the PC terminal as an example to introduce the system. The development of the system platform based on the virtual display of cheongsam mainly involves five steps: model introduction, model and special effect design, scene interactive development, collision detection, and export application.

\subsection{Model import}

Using MAYA and 3D MAX to build scenes, cheongsam, characters and so on, we completed them altogether. Due to import the model into Unity 3D will result in material loss. For this problem, we need to import material again to achieve the matching state between material and model. The static model can't satisfy the scenario design and the animation parts, for example the character who is walking in the scene needs to be imported and adjusted.

\subsection{Model and special efficiency design}

Model design and special efficiency design are one of the most important parts after the introduction of models. The advantages and disadvantages of models and special efficiency design are important criteria for determining whether the scene environment is contagious.

Model design is based on model reorganization and disassembly. During the roaming, model resolution is to prepare for later interaction settings, especially the interaction between the visitors and the interaction in the environment when they experienced the Virtual Display System. Good model resolution and reorganization can provide efficient and high-quality scenario models for script operation.

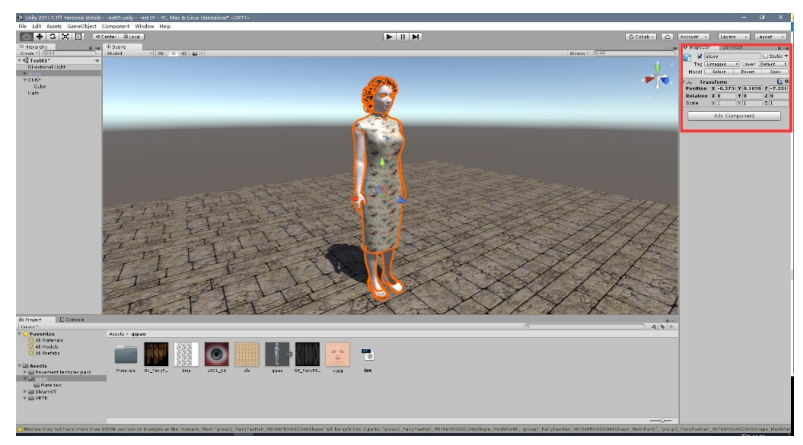

Figure 6. Special efficiency personage binding by Unity 3D

The Virtual Display System of cheongsam mainly involves special effects such as "light", "sound", "reflection" and so on. The most operation is the fade in and out function of the model of the cheongsam in the exhibition hall. Therefore, figure 6 shows that a special binding is carried out on the cheongsam character model, and the script is run to control the model.

\subsection{Interactive development of scene}

Scene interactive development mainly involves three main parts. One is the scene animation call, the two is the rigid body attribute to the object, and the three is collision detection. This is a step that must be completed before exporting the Virtual Display System of cheongsam.

Scene animation into Unity 3D still needs to be animated and invoked, which can enhance its dynamic sense. Secondly, adding rigid body can make the visitor experience the effect of force and enhance the authenticity of the scene.

Collision Detection is a necessary step after completing the Virtual Display System. It is to check whether the experienced person has penetrated and other accidents in the roaming system. Collision Detection between models also prevents visitors from 
appearing in virtual scenes without interaction events. The collision detection process, as shown in figure 7 shows that the collider, a Sphere Collider, is run with the Unity 3D to detect the collision experiment of the Virtual Display System.

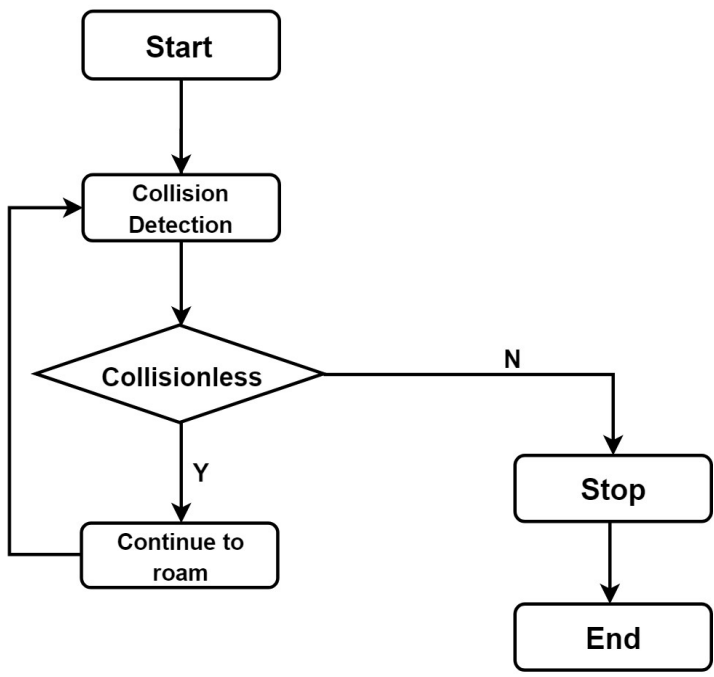

Figure 7. Flowsheet of Collision Detection

\subsection{Unity 3D output form}

There are three types of virtual reality: PC terminal, mobile terminal and all-in-one. In the Cheongsam Virtual Display System, PC and mobile terminal which is convenient and fast with excellent experience will be adopted. After determining two types of forms, the model is imported into Unity $3 \mathrm{D}$ before the corresponding form is set out for the output forms, and the products are exported in two times.

The mobile version is mainly linked with WEB to the public and APP of Shanghai Museum of textile and costume, which enables visitors to experience the virtual display of cheongsam at home. As figure 8 shows, it's pretty convenient to enjoy the view by phones.

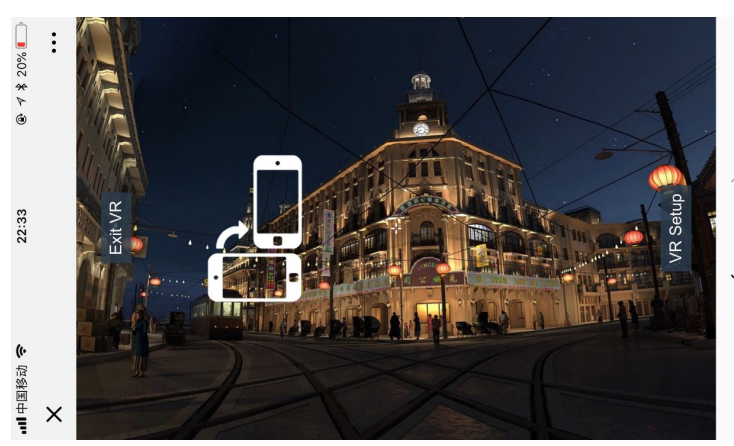

Figure 8. Virtual Display System by mobile
The PC version is mainly set up in the exhibition hall, providing the visitors with an experience of ultrahigh immersion, interaction and imagination. Shanghai Museum of textile and costume mainly uses HTC VIVE as the device, and the visitors can control the roaming path and interact with it by means of a handle. As figure 9 shows, we also can experience the Virtual Display System in the museum by desktop computer and Immersive headgear.

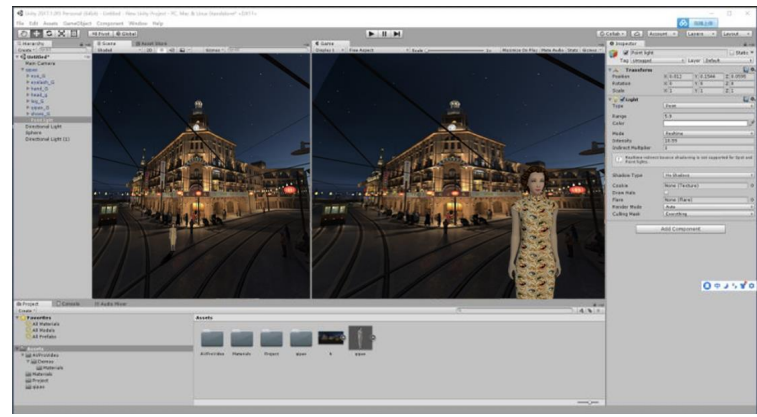

Figure 9. Virtual Display System by PC

\section{Conclusion}

During the construction of the Virtual Display System of cheongsam, the elements of the whole virtual system are built by modelling the character, cheongsam and environmental scenes. Because of the particularity of the museum, the virtual cheongsam is displayed in the modelling and later design, with the help of historical data to design characters and scenes. Following the principle of resilience, we can truly display the cheongsam in the living environment of the Republic of China. Text, audio, sound efficiency and other elements to optimize the visitors' sensory experience of the virtual system. Finally, all components are assembled in Unity $3 \mathrm{D}$ to optimize and adjust and release two types of experience, mobile version and PC version.

\section{References}

1. Figure 4 from http://book.kongfz.com/22912/673851891/

2. QP. Zhao. Science China. J.02 (2009) (to be published) 